\title{
LOS CONCEPTOS DE BIOLOGIA ADQUIRIDOS EN EL PROCESO DE APRENDIZAJE
}

GIORDAN, A.

Laboratorio de didáctica y epistemologia de las Ciencias. Universidad de Ginebra.

(Versión de Maria Jesús Caballer Senabre)

SUMMARY

By using instances from Biology, the function of preconceptions and their place within the learning process is analyzed.

\section{INTERES DIDACTICO DE LAS PRECON- CEPCIONES}

Actualmente, el alumno es el «presente-ausente» en el proceso educativo ya que el profesor no tiene ni las claves, ni los medios para integrar al alumno en los procedimientos didácticos; en efecto, el profesor no puede orientar los procesos de comprensión o de aprendizaje del alumno pues le faltan, por su formación insuficiente, las técnicas de análisis adecuadas. Siendo así podemos comprender la separación considerable entre el maestro, que concibe sus clases con su propia lógica de adulto especializado, y el alumno que intenta hacerse una idea de estos conocimientos a través de sus representaciones. El alumno, frente a un problema, dispone de una representación mental de la misma aunque esta no puede aportar elementos de explicación al problema que tiene ante sí (Giordan, 1976).

Tradicionalmente, el profesor está muy poco preocupado por el marco de referencias del alumno, sea porque sus razonamientos van a reemplazar dicho marco o porque desconfía de la capacidad del alumno. Esta ignorancia respecto al alumno tiene raices profundas y se mantiene porque se busca analizar el pensamiento del mismo plagiando los razonamientos y los marcos de referencia del ensef̂ante mientras que hay muy poca preocupación por lo que el alumno aprende. Bachelard puso en evidencia la dificultad de cambiar de modelo, de substituir las interpretaciones inmediatas del alumno por las representaciones de la ciencia (Bachelard lo justificaba entonces por el hecho de que «las explicaciones cientificas no tienen éxiton y por el hecho de que (era necesario cambiar el nivel cultural») (Giordan, 1982).
Cuando el individuo aprende, no llena jamás un vacio sino que substituye poco a poco representaciones «intuitivas" que expresan la visión que los alumnos, e incluso los adultos, tienen del mundo que les rodea. Estas representaciones son traducciones particulares de la realidad que no resultan de un análisis riguroso: son imágenes que se apoyan en analogias artificiales, en las que los términos no se definen de forma univoca y que son difícilmente comunicables. No son puestas jamás en cuestión por sus autores, ni se observan en ellas las contradicciones internas que aparecen cuando se les analiza desde nuestra posición de adulto y de especilista. A pesar de esto que acabamos de decir, las representaciones no son un juego gratuito para los alumnos, son coherentes para ellos y tienen un valor significativo en función de sus modelos de pensamiento específicos. Además es muy difícil expresar cualquiera de estas representaciones, no bastan las palabras y por eso escapan a la confrontación con la realidad y con las representaciones que puedan tener otros. Estos esquemas mentales no pueden cambiar profundamente o ser desplazados por las explicaciones externas de la lógica del enseñante; si no se les tiene en cuenta podemos propiciar la coexistencia en los alumnos de dos sistemas explicativos paralelos: uno será utilizando en las situaciones de clase estrechamente orientada por el profesor, el otro resurgirá con tenacidad cuando la situación sea menos "escolar» (fig. 1).

Un cierto número de trabajos, por otra parte puntuales, se han desarrollado para reutilizar estas representaciones con fines didácticos. En biología sobre la nu- 


\section{figura I}

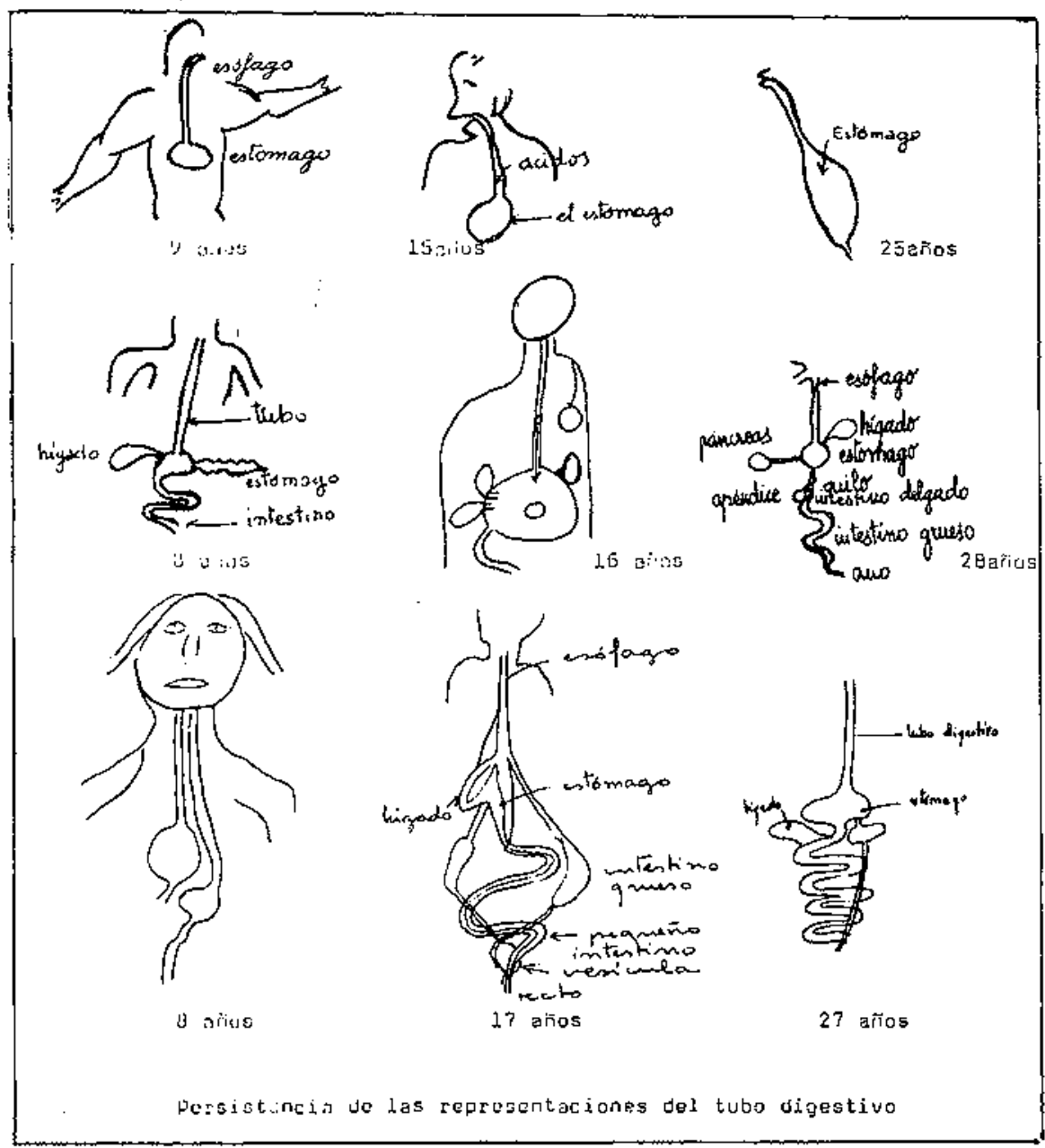

trición, crecimiento, desarrollo, formas de respiración.... en física sobre electricidad, magnetismo, estructura de la materia, etc.

Parece que estos trabajos han tenido el mérito de atraer la atención sobre estos aspectos de la investigación didáctica, el interés por conocer las etapas y formas de construcción del saber en el alumno; pero han reintro" ducido, por otra parte, la importancia del profesor, en oposición a los movimientos no directivos de los años 70. El maestro no puede intervenir con una cierta eficacia nada más que si está atento al alumno, si toma en cuenta sus representaciones y sus procesos de apren " dizaje.

\section{FUNCION DE LAS PRECONCEPCIONES Y SU LUGAR EN EL PROCESO DE APREN- DIZAJE}

Los «conceptos» tal como nosotros los reconocemos, son a la vez el producto y el proceso de una actividad de construcción mental de la realidad. Esta elaboración se efectúa con seguridad a partir de las informa ciones que el discente recibe por medio de sus senti- dos, pero también a través de las relaciones que mantiene con otros, individuos o grupos, en el curso de sus vida y que quedan grabados en la memoria. Estas in. formaciones son codificadas, organizadas y categorizadas en un sistema cognitivo global y coherente, en relación con sus preocupaciones y con la utilización que haga de las mismas.

Al mismo tiempo los conceptos anteriores filtran, escogen y elaboran las informaciones recibidas y pueden ser completados, limitados o transformados, lo que da lugar a nuevos conceptos.

Esta actividad de construcción mental de lo real se efectúa según modelos psicológica y socialmente determi nados y son interdependientes. La realidad es la fuente a partir de la cual el sujeto concibe, pero esta realidad es cercada, recortada, descodificada y explotada en función del marco de referencia y de las operacio. nes del que aprende, lo que le permite construir un tipo de lectura aplicable a su entorno. Este instrumento de análisis le lleva a organizar el mundo, o un aspecto del mundo, de tal manera que pueda comprenderlo, desenvolverse en él, adaptarse o evadirse, al menos a un cierto nivel. 
Trataremos de explicar al máximo nuestra idea sobre la construcción y las funciones de estas concepciones que nosotros llamaremos «constructos».

Comenzaremos por enumerar los componente de un constructo que designaremos con las siglas $\mathrm{P}, \mathrm{M}, \mathrm{O}, \mathrm{R}$ y $S$ :

$P=$ Problema y conjunto de cuestiones que provocan el desarrollo de este concepto. (Es un poco el motor de la actividad intelectual).

$\mathrm{M}=$ Marco de referencia: conjunto de conocimientos periféricos utilizados por el sujeto para formular su concepto (son las otras representaciones que pueden in. terferir con la principal).

$\mathrm{O}=$ Operaciones mentales: conjunto de operaciones intelectuales que el sujeto es capaz de hacer y que le permiten producir y utilizar su concepto (son las invariantes operatorias).

$\mathrm{R}=$ Red semántica: es la que a partir del marco de referencia y de las operaciones mentales, permite dar un sentido al concepto (el sentido del constructo aparece a partir de las relaciones establecidas entre los diferentes conceptos - principal y periféricos).

$\mathrm{S}=$ Significantes: conjunto de signos, trazos y símbo los utilizados en la construcción de! concepto.

Un ejemplo va a permitir que nos expliquemos mejor:

Cuando, al nivel universitario, se trabaja sobre el concepto de memoria, el marco de referencia puede ser representado de la siguiente manera (fig. 2).

\section{figura 2}

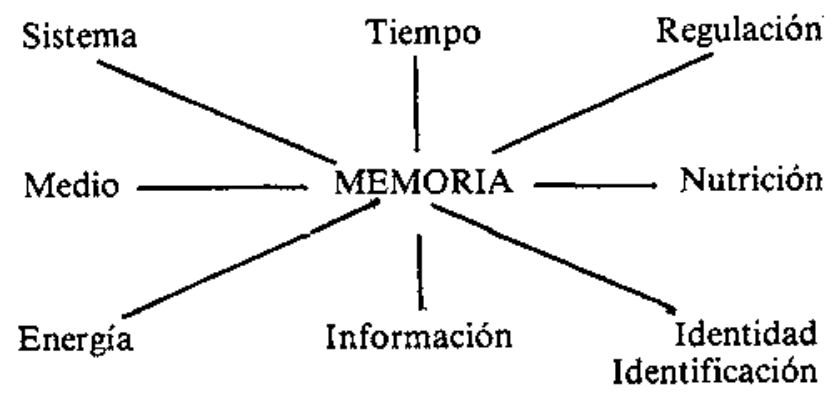

Este esquema está constituido por el conjunto de las otras nociones necesarias para comprender el estado actual de este concepto. Es lo mismo para los conceptos que elaboran los alumnos: si la fecundación es «un huevo resultante del encuentro de un espermatozoide $y$ de un ovulo" y eso corresponde al punto de partida del desarrollo del ser vivo, esta visión lleva aproximadamente al siguiente marco de referencia (fig. 3). figura 3

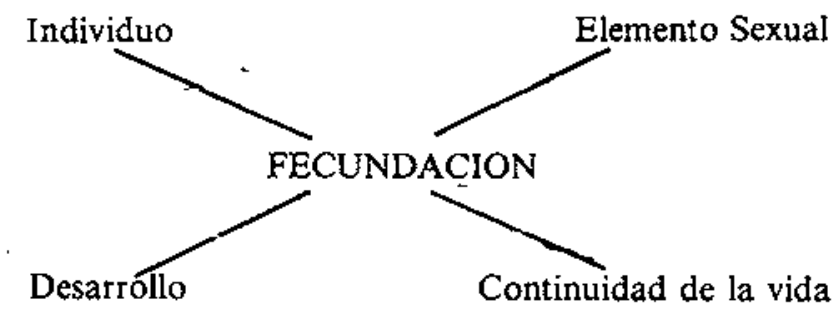

No todos los elementos a los cuales se recurre están in cluidos en el «construct» porque no están ligados en. tre ellos de una forna natural. En este ejemplo, el que aprende tiende a responder a una cuestión (incluso si esta no está explicitada): « $_{i}$ a partir de qué se fabrica un individuo?; el alumno ha establecido, entre elementos de su marco de referencia, un cierto número de operaciones mentales, ha definido un campo de comprehensión (en este caso serian mecanismos de transformación, movimientos de gametos, papel de los espermatozoides no fecundantes) y una cierta extensión (se limita a los animales con organos diferenciados, para las plantas no sabe muy bien qué ni cómo). Todo esto junto adquiere un sentido propio, este conjunto de re. laciones constituyen el concepto del sujeto, es a esto a lo que denominaremos red semántica. Lo que la distingue del marco de referencia es que a partir de un mismo marco de referencia es posible obtener conceptos distintos. Basándonos en este mismo caso podríamos comentar que cuando un alumno dice: «se pueden producir individuos mediante el encuentro de dos simientes", se está apoyando sobre el mismo marco de referencia que el anterior, pero a partir de una cuestión inicial distinta, en relación con el medio agrícola en el que se desenvielve, su problema no es el mismo: busca obtener - - por técnicas de campo- individuos de la misma «especie» que la de sus padres. El campo de comprehensión en este último caso está determinado fundamentalmente por una cuestión práctica en relación con los animales de cría en granja.

La posibilidad de que los conceptos sean perfilados en el pensamiento depende necesariamente de los plantea. mientos que tenga el individuo puesto que cualquier representación es inviable si no es con referencia a un problema que se plantea el sujeto (este problema es frecuentemente implicito). Podemos decir también que los conceptos no son simples imágenes o representaciones mentales, son más bien índices de un modelo, un tipo de discurso intelectual, en respuesta a un problema o a una serie de problemas.

Teniendo en cuenta esto proponemos que la elaboración mental de un concepto depende de la interacción de tres parámetros: el marco de referencia, las inva riantes operatorias y la red semántica. 
Llamaremos marco de referencia al conjunto de conocimientos anteriormente integrados por el individuo que, unidos, dan un sentido al nuevo concepto. Es a menudo este marco de referencia el que le va a llevar a formularse preguntas e intentar buscar las respuestas.

Las invariantes operatorias constituyen el conjunto de operaciones mentales que van asociadas a la construcción mentai del concepto, que permiten hacerlo funcionar y también permiten transformarlos a partir de las informaciones recibidas.

La red semántica es la estructura que da un sentido al conjunto a partir de las operaciones y los conocimien. tos puestos en juego. Constituye una especie de red en la que los nudos representan el marco de referencia y los enlaces (entre cada nudo) pueden asimilarse al papel de las operaciones mentales.

Dentro de los significantes incluimos el lenguaje y el conjunto de símbolos y de otras formas de representación (lenguaje natural, matemático, esquemático,...) que son utilizados para explicar el concepto adquirido.

Hemos separado estos diferentes aspectos con el fin de analizarlo mejor: en realidad, funcionan de forma interdependiente y son parcialmente superponibles, como muestra el modelo siguiente (fig. 4).

Una de las primeras funciones atribuible a los conceptos adquiridos reside en la conservación de un conocimiento al que más tarde, cuando sea requerido, tendremos acceso directo. Pero esta función no se desarrolla como una acumulación de simples recuerdos: la información así adquirida es -en efecto- conservada pero puede ser reutilizada en situaciones nuevas, por ejemplo en la elaboración de un itinerario en un espacio complejo o en el control del funcionamiento de una máquina.

Otra función importante es, sin duda, la de la sistematización. Por ejemplo: la taxonomía aplicada al mundo vivo o la tabla de clasificación de los elementos pe riódicos de Mendéléeff son representantes de un conjunto de hechos y relaciones que se establecen entre ellos. Vemos cómo esta forma de representación asegura la sistematización de un corpus de datos. Consideraciones similares valen para los organigramas, o para los diagramas tales como los que ilustran el funcionamiento de un ecosistema.

Las representaciones cognitivas no deben pues ser únicamente interpretadas como colecciones de informaciones o como los elementos de un stock informativo simplemente destinado a consultas ulteriores. Tomando como punto de partida los conceptos adquiridos, el sujeto ha de ser capaz de resolver problemas nuevos, realizar actividades distintas a las del pasado, con cebir nuevos algoritmos de conducta, abordar proyectos originales para él y encontrar otros objetivos que alcanzar: esta función corresponde a una movilización de lo adquirido. A partir de la experiencia cada uno de nosotros constituye en su interior una especie de «rejilla de análisis» de la realidad; los «constructos» nos permiten comprender el mundo que nos rodea, abordar situaciones nuevas y actuar (fig. 5).

Los conceptos previos del que aprende se situan en el mismo centro del problema del aprendizaje ya que estos conceptos participan en el juego de relaciones exis. tente entre las informaciones de las que dispone un in-

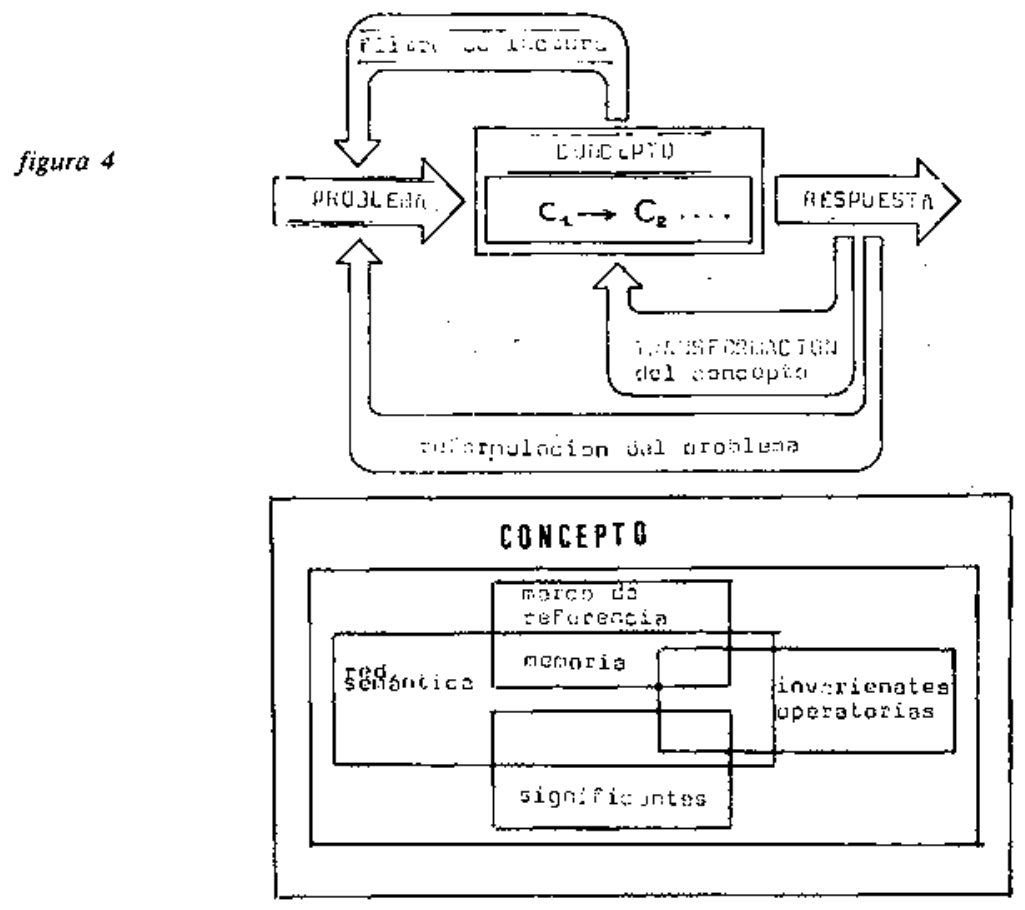


figura 5

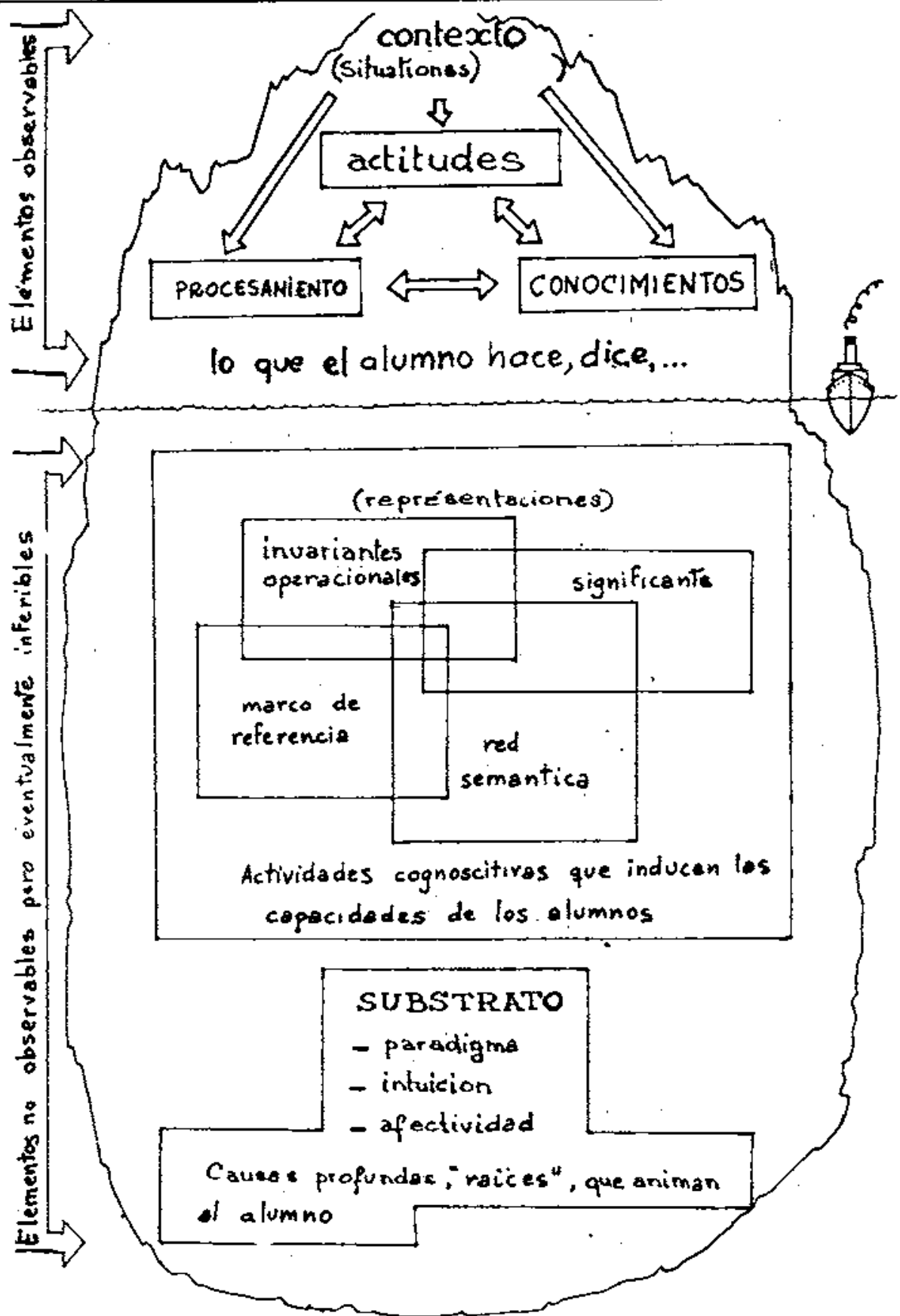

dividuo y las que podrá encontrarse a lo largo de su existencia; es sobre estos elementos que se elaborarán sus nuevos conocimientos y, por tanto, sus conductas futuras.

El profesor debe de conocer estos procesos e intentar incidir en ellos; si no lo hace asi su acción educativa corre el riesgo de no ser eficaz.

\section{ELEMENTOS PARA UN TRATAMIENTO DIDACTICO DE LAS PRECONCEPCIONES}

El conocimiento de los conceptos que ha conformado el alumno plantea toda una serie de problemas:
¿Qué hacer con estas representaciones de los alumnos en el proceso educativo?

¿Cómo apoyarse en ellos para ayudar a los alumnos a apropiarse de algunas parcelas del saber científico?

¿Sus representaciones pueden ser consideradas como una primera explicación, a falta de algo mejor, para llevar a los alumnos a la adquisición de un concepto?

¿Deben tomarse como errores que se han de eliminar pura y simplemente?

¿Pueden ser rectificados por una pedagogia que los tenga en cuenta para modificarlos haciendo que el alumno razone los factores por los cuales ha llegado a cons- 
truir esa representación y de esta manera la rectifique?

¿Pueden someterse a una confrontación con representaciones contradictorias o a una confronfación con la realidad que permita un tanto experimental o incluso se pueden confrontar con informaciones aportadas por libros o profesores para inducir la adquisición del con* cepto más aproximado a la realidad?

Según nos muestran las investigaciones actuales es difícil de decidir, no podemos resumir una estrategia pedagógica eficaz en todo momento. Entonces ¿cómo avanzar?

Primeramente, los conceptos previos no pueden ser considerados como un fin de la investigación educati. va en sí mismos. No observamos modificaciones espontáneas que permitan el paso directo de las representaciones subjetivas al conocimiento científico. La evolución de los conceptos adquiridos por el alumno es lenta, necesita mucho tiempo y detenerse en muchos puntos: algunos conceptos que dan sentido a otro concep. to que estamos estudiando deben ser modificados al mismo tiempo y para comprender bien este último. (Por ejemplo, en relación con los mohos surgen también preguntas y precisiones sobre el concepto de ser vivo, generación espontánea, aire...). En un aprendizaje metodológico deben desarrollarse en paralelo las variables permanentes que sean necesarias.

Por lo tanto las representaciones de los alumnos no deben ser tomadas en cuenta antes de iniciar una fase educativa, únicamente para prepararla, sino sobre todo durante esta. Estas representaciones deben de ser enfren. tadas a otras representaciones, a otros fenómenos, a experiencias sensibies. El profesor debe de ser uno de los elementos de esta confrontación permitiendo y aportando informaciones contradictorias, o proponiendo st. tuaciones para comprobar todas las representaciones. Con sus aportaciones debe de favorecer la mutación, es decir provocar una ruptura referente a las represen-

\section{REFERENCIAS BIBLIOGRAFICAS}

GIORDAN, A., 1976, Rien ne sert courir il faut partir à point (These Université Paris 1976) publié dans Une pédagogie pour les sciences expèrimentales (Centurion, 1978). (Traducido en castellano: Ed. Pablo del Río).

GIORDAN, A., 1982, L'élève et/ou les connaissances scientifiques. (Ed. Lang).

GIORDAN, A. et DE VECCHI, G., 1987, Les origenes du taciones inmediatas y favorecer una reorganización de los campos de conocimiento.

Consideramos, después del análisis riguroso de nues" tras investigaciones, que los "pre-conceptos" de los alumnos nos deben servir como indicadores que per. mitan a los profesores autorregular la práctica peda gógica. Esto nos conduce a proponer las bases de una pedagogía diferenciada, dependiente de los problemas presentados, de los objetivos perseguidos y sobre todo de los alumnos: es decir una pedagogia no tipificada que presenta características diversas según las circunstancias. A veces una simple indicación, una flecha, es suficiente para ayudar al alumno, en otros momentos se le debe de hacer pasar por fases de una investigación importante. Estas intervenciones no están decididas "a priori», depende de los interrogantes que se plantee el alumno, del marco de referencia que tenga.

Nuestros trabajos más recientes se centran en esta di. rección, ensayando un desarrollo paralelo de las estrategias pedagógicas que tienen éxito en el proceso educativo y mediante una aproximación a las representa. ciones de los alumnos. Entonces estas servirán como elemento de diagnóstico que permitiría conocer mejor el saber y la práctica adquirida por el alumno e inter. ferir en ellos. Estas representaciones permitirían comenzar a estructurar este rompecabezas pues si el espíritu del alumno es a menudo opaco para el adulto no es porque tenga piezas de menos sino porque estas piezas se combinan de diferente forma a como ocurre en los adultos, porque responden a otro tipo de pensamiento que el adulto superó hace mucho tiempo, pero al que puede ser útil reaprenderlo si tiene como objetivo aportarle mayor conocimiento.

Así los trabajos sobre los conceptos adquiridos por los alumnos permiten una visión nueva. La relación pedagógica se transforma dándole un lugar al que aprende: el alumno, pues sólo este último puede construir su saber a condición de que se le ayude a hacerlo.

savoir. Des conceptions atux concepts (Delachaux et Niestłé: Neuchâtel).

Nota: Una bibliografia amplia sobre los preconceptos en Biología puede ser obtenida solicitandola a:

A. Giordan, LDES,

24 Rue du General Dufour

1211, Ginebra M. (Suiza) 